\title{
Full-Potential Optical Calculations of Lead Chalcogenides
}

\author{
ANNA DELIN, ${ }^{1}$ P. RAVINDRAN, ${ }^{1}$ OLLE ERIKSSON, ${ }^{2}$ \\ J. M. WILLS ${ }^{2}$ \\ ${ }^{1}$ Department of Physics, Uppsala University, P.O. Box 530, S-75121 Uppsala, Sweden \\ ${ }^{2}$ Center for Materials Science and Theoretical Division, Los Alamos National Laboratory, Los Alamos, \\ New Mexico 87544
}

Received 29 June 1997; revised 30 September 1997; accepted 24 October 1997

\begin{abstract}
We report on ab initio calculations of the optical properties of the lead chalcogenides $\mathrm{PbS}, \mathrm{PbSe}$, and $\mathrm{PbTe}$ performed with a relativistic full-potential linear muffin-tin orbital method within the local density approximation. Our calculated spectra are in excellent agreement with recent ellipsometry measurements. The origin of the peaks in the spectra is discussed, as well as the effects of increasing the chalcogen atomic number. @ 1998 John Wiley \& Sons, Inc. Int J Quant Chem 69: 349-358, 1998
\end{abstract}

Key words: density functional theory; full-potential linear muffin-tin orbital method; optical properties; lead salts

\section{Introduction}

$\mathbf{T}$ he IV-VI semiconductors $\mathrm{PbS}, \mathrm{PbSe}$, and $\mathrm{PbTe}$, generally known as lead salts, have been the subject of a vast amount of theoretical and experimental work during the past decades, motivated in part by their technological usefulness, but also by their unusual and interesting physical properties among semiconductors. These include soft zone-center transverse optical phonon

Correspondence to: A. Delin.

Contract grant sponsors: Swedish Research Council for Engineering Sciences; Swedish Natural Science Foundation; Swedish Materials Consortium No. 9. frequency, ferroelectricity, large optical dielectric constant, and a narrow fundamental energy gap whose temperature coefficient $d E_{g} / d T$ is positive $[1,2]$. The lead salts are of importance in infrared detectors, in light-emitting devices, and more recently as infrared lasers in fiber optics, as thermoelectric materials, in solar energy panels, and in window coatings [3-6].

All three lead salts are stable in the rocksalt structure at ambient temperature and pressure, with lattice parameter $5.94 \AA$ for PbS, $6.13 \AA$ for PbSe, and $6.46 \AA$ for PbTe. Furthermore, they all have a direct minimum energy band gap of around $0.3 \mathrm{eV}$ at the $L$ point in the Brillouin zone (BZ).

Very recently, spectroscopic ellipsometry (SE) measurements for a rather wide energy range were 
reported for PbSe and PbTe; see Refs. [7] and [8], respectively. High precision experimental determination of the dielectric function of $\mathrm{PbSe}$ in a more narrow energy range have also been performed by Globus, Olesk, and Olesk [9]. They used parallel measurements of the optical transmission and reflection on thin films of PbSe. The big advantage of the SE technique, and other parallel measurement techniques, is that both the real and imaginary parts of the dielectric functions are obtained directly on a wavelength-by-wavelength basis, without having to employ multiple, nonsimultaneous, measurements or resort to Kramers-Kronig analysis. This means that several sources of error can be avoided, for instance the uncertainty in the absolute amplitude caused by the extrapolations necessary for the Kramers-Kronig transformation of experimental reflectivity spectra. These attractive features make comparisons with SE measurements, and probably also with other types of parallel measurement techniques, ideal for evaluating how well our theoretical method succeeds in reproducing optical spectra. The present calculational method has previously been used, with positive results, to calculate the optical spectra of several systems, e.g., $\mathrm{ZrN}$ and related systems [10] and $\mathrm{CeN}$ [11].

The electronic structure of the IV-VI semiconducting compounds have been quite extensively studied in the past. However, no ab initio calculation of the optical properties have yet been performed for these materials. The band structures of the lead salts have been calculated using many different methods such as the empirical pseudopotential method $[12,13]$, the augmented plane wave method [14], the orthogonalized plane wave method [15], tight binding [16], and linear combination of atomic orbitals [17]. All of them give results with several features in common, for example, the direct gap at the $L$ point in the Brillouin zone. The optical spectra of the lead salts have previously been calculated using the empirical pseudopotential method [13].

\section{Details of Calculations}

In the density functional method used in the present study, the Kohn-Sham equations [18] are solved for a general potential without any shape approximation [19]. For the density functional, we used the local density approximation (LDA) in the
Hedin-Lundqvist parametrization. We also tested the more advanced Perdew-Wang generalized gradient approximation (GGA) [20] to the density functional. However, this more advanced scheme caused virtually no change of the optical properties relative to the LDA. In the present method, space is divided into nonoverlapping spheres (called atomic or muffin-tin spheres [21]) surrounding each atomic site, and an interstitial region. The basis functions used are energy-independent Bloch functions, whose construction is somewhat different in the atomic spheres and in the interstitial. Together with the variational principle, this leads to the secular equations becoming linear in energy and reducing to generalized eigenvalue equations, which can be solved by diagonalization. In the interstitial region, the charge density is slowly varying, and the natural basis is plane waves or Hankel and Neumann functions. A basis function in the interstitial is therefore expressed as a Bloch sum of Hankel and Neumann functions which in turn is represented as a Fourier series. Formally, a basis function in the interstitial is defined by the Bloch function of solutions to the spherical Helmholtz equation with nonzero kinetic energy $\kappa^{2}$, or a linear combination of such solutions for different kinetic energies. The Fourier representation of this basis function is taken from the Fourier series of a function matching the basis in the interstitial region but not inside the atomic spheres, a so-called pseudowave function, whose exact shape inside the muffin-tin sphere is of no importance for the final solution as long as it is continuous and differentiable at the sphere boundary and matches the true basis function in the interstitial. It must also have zero slope at the origin of each sphere.

Inside the atomic spheres, where the charge density varies rapidly, the basis functions are Bloch functions of radial functions times spherical harmonics. The radial part of a basis function is constructed from the numerical solutions $\phi_{L}\left(E_{\nu}, r\right)$ of the radial Schrödinger equation in a spherical potential at the fixed energy $E_{\nu}$, and their energy derivatives $\dot{\phi}_{L}\left(E_{\nu}, r\right)$. Here, the index $L$ stands for a collection of quantum numbers: the principal quantum number $n$, the orbital quantum number $l$, azimuthal quantum number $m$, and the kinetic energy $\kappa^{2}$. The treatment of the entire basis set within one single energy panel allows all states, including the pseudocore states, to hybridize fully with each other. The method is linear, i.e., the basis functions are constructed by expanding 
around fixed energies $E_{\nu}$. Such an approach normally leads to linearization errors for higher lying bands. However, this difficulty is to a large extent overcome in the present calculation by allowing for an extended basis set where every orbital is described by several kinetic energies in the interstitial region.

The expressions for the crystal wave functions in the muffin-tin spheres are matched to the interstitial crystal wave function at the sphere boundaries so that the total crystal wave function becomes continuous and differentiable in all space.

The potential used for solving the radial Schrödinger equation above is obtained from the charge density by solving Poissons equation. In the first iteration, this density is taken as overlapping atomic charge densities. A new charge density is then constructed from the eigenvectors obtained through the variational procedure, and a new solution is obtained. The procedure can then be repeated until some criterion for self-consistency is met.

The integration over the Brillouin zone in the ground-state calculation was performed using the special point sampling [22]. Furthermore, spin-orbit coupling is included in all calculations presented here.

We adopted the dipole approximation in our optical calculations, i.e., the momentum transfer from the initial state to the final state was neglected. The dielectric function was calculated in the momentum representation, which requires matrix elements of the momentum $\mathbf{p}$ between occupied and unoccupied states. Thus, the components of the imaginary, or absorptive, part of the dielectric function, $\epsilon_{2}^{i j}(\omega)$, was calculated from

$$
\begin{aligned}
\epsilon_{2}^{i j}(\omega)= & \frac{4 \pi^{2} e^{2}}{V m^{2} \omega^{2}} \\
& \times \sum_{\mathbf{k} n n^{\prime} \sigma}\left\langle\mathbf{k} n \sigma\left|p_{i}\right| \mathbf{k} n^{\prime} \sigma\right\rangle\left\langle\mathbf{k} n^{\prime} \sigma\left|p_{j}\right| \mathbf{k} n \sigma\right\rangle \\
& \times f_{\mathbf{k} n}\left(1-f_{\mathbf{k} n^{\prime}}\right) \delta\left(E_{\mathbf{k} n^{\prime}}-E_{\mathbf{k} n}-\hbar \omega\right),
\end{aligned}
$$

where $e$ and $m$ is the electron charge and mass, respectively, $\omega$ is the frequency of the incoming electromagnetic radiation, $V$ is the volume of the unit cell, $\left(p_{x}, p_{y}, p_{z}\right)=\mathbf{p}$ is the momentum operator, $|\mathbf{k} n \sigma\rangle$ the crystal wave function, corresponding to eigenvalue $E_{\mathbf{k} n}$ with crystal momentum $\mathbf{k}$ and spin $\sigma$. Finally, $f_{\mathbf{k} n}$ is the Fermi distribution function ensuring that only transitions from occu- pied to unoccupied states are counted, and $\delta\left(E_{\mathbf{k} n^{\prime}}-E_{\mathbf{k} n}-\hbar \omega\right)$ is the condition for total energy conservation.

The evaluation of the matrix elements in the above equation involves integration in real space. This integration is done over the muffin-tin spheres and the interstitial separately. Inside the muffin-tin spheres, it is natural to use spherical coordinates for the integration. The integral can be expressed as a radial function times the angular integral. Integration over the angular coordinates in real space, assuming the electric dipole approximation, gives rise to the electric dipole selection rules, i.e., that some of the angular integrals are exactly zero. The nonzero angular integrals can be expressed exactly using Gaunt coefficients and spherical harmonics. The radial integral is, of course, intrinsically numerical and is evaluated by performing the differentiation numerically, followed by integration. The volume integral in the interstitial can be rewritten as a surface integral over the muffintin spheres using Green's formula. Explicit expressions regarding this integration can be found in Ref. [23].

The summation over the Brillouin zone is calculated using linear interpolation on a mesh of uniformly distributed points, i.e., the tetrahedron method. Since all information in the problem is contained within the irreducible wedge of the Brillouin zone (IBZ), Eq. (1) is evaluated in the IBZ, giving $\boldsymbol{\epsilon}_{2}(\omega, \mathrm{IBZ})$. The total imaginary part of the dielectric function $\boldsymbol{\epsilon}_{2}(\omega)$ is then obtained by performing all symmetry operations on $\boldsymbol{\epsilon}_{2}(\omega, \mathrm{IBZ})$, summing up and dividing by the number of symmetry operations, i.e.,

$$
\boldsymbol{\epsilon}_{2}(\omega)=\frac{1}{N} \sum_{i=1}^{N} \mathscr{S}_{i}^{T} \boldsymbol{\epsilon}_{2}(\omega, \mathrm{IBZ}) \mathscr{S}_{i},
$$

where $N$ is the number of symmetry operations and $\mathscr{S}_{i}$ represents the $i$ th symmetry operation. When the crystal symmetry is cubic, as in the present case, the dielectric tensor becomes diagonal, and may therefore be represented by a scalar, $\epsilon(\omega)$ instead of $\epsilon(\omega)$ with components $\epsilon^{i j}(\omega)$. We will use this simplified notation in the following.

The real, or dispersive, part of $\epsilon(\omega)$, i.e., $\epsilon_{1}(\omega)$, was obtained by Kramers-Kronig transformation of the imaginary part over the energy range of $0-3$ Ry. This energy interval was seen to give convergence in the Kramers-Kronig transformation, as 
checked by performing the transformation twice and comparing the result with the untransformed original spectrum.

\section{Results and Discussion}

Before turning to the optical results, we first discuss the electronic structure and bonding mechanism in these compounds.

The total state densities for all three compounds are shown in Fig. 1. As seen in this figure, the total state densities (DOS) are quite similar for all three compounds, with some smaller differences in the details. The first structure encountered in the DOS, if we start from the left, is a narrow peak centered around $-13 \mathrm{eV}$ for $\mathrm{PbS}$ and PbSe and $-11 \mathrm{eV}$ for $\mathrm{PbTe}$. This peak consists almost entirely of chalcogen $s$ states (see Fig. 2) where the partial state densities (PDOS) are shown. Furthermore, this peak corresponds to the lowest lying band in Figure 3 , and its width originates mainly from the region around the $\Gamma$ point in the $\mathrm{BZ}$, since only there the dispersion of this band is appreciable.

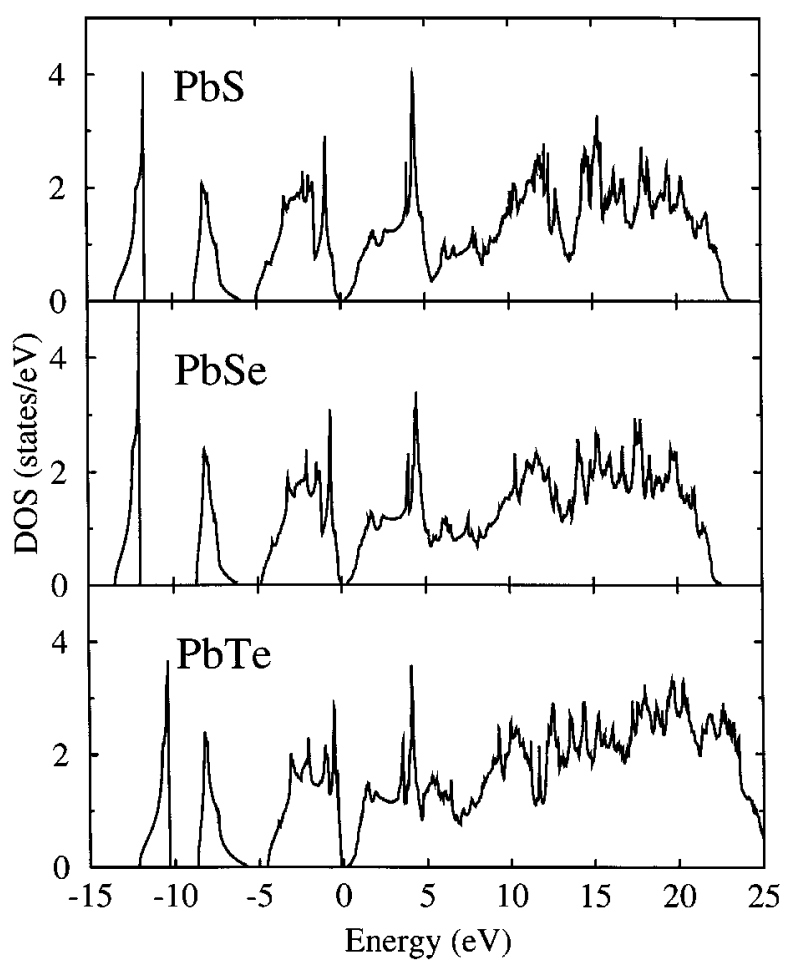

FIGURE 1. Total DOS (in states per electron volt and formula unit) for $\mathrm{PbS}, \mathrm{PbSe}$, and $\mathrm{PbTe}$. The Fermi energy is at zero.
The narrow structure centered at $7 \mathrm{eV}$ below the Fermi level consists predominantly of $\mathrm{Pb} 6 s$ states but also of chalcogenide $s$ and $p$ states, as is apparent from Figure 2. This structure is highest in $\mathrm{PbTe}$ and smallest in PbS and corresponds to the rather flat, next lowest lying band shown in the band structures in Figure 3. From the band structures we also see that the width of this peak originates from the disperson in the region near $\Gamma$, and also to some extent from the region around $X$. In all three compounds, the structure at $-7 \mathrm{eV}$ is well separated from the next broader structure, situated between $-5 \mathrm{eV}$ and the Fermi level. This structure consists mostly of chalcogen $p$ states but also to some extent of $\mathrm{Pb} 6 s$ and $6 p$ states; see Figure 2. The $d$ contribution to this structure is very small, just as for the lower, s-dominated, peaks. In $\mathrm{PbS}$ and $\mathrm{PbSe}$, there is a valley in this structure at about $1 \mathrm{eV}$ below the Fermi level. In $\mathrm{PbTe}$, this valley is almost gone. The k-resolved origin of this difference can be elucidated from Figure 3, where the band structures of all three compounds are shown. Comparing the band structures around the $L$ point about $1 \mathrm{eV}$ below the Fermi level, we see that two bands are degenerate in this region for $\mathrm{PbS}$ but not so for $\mathrm{PbTe}$. In PbSe, they are almost degenerate. Continuing upward in energy, we see that all three compounds have a distinct energy gap at the Fermi level. Also, from the partial state densities in Figure 2, it is seen that just below the Fermi level, the bands are dominated by chalcogen $p$ states, with some contribution coming from the $\mathrm{Pb} 6 p$ states. In the region above the Fermi level, $p$ states also dominate, but the roles played by the $\mathrm{Pb} 6 p$ states and chalcogen $p$ states are roughly reversed. Thus, just above the Fermi level the $\mathrm{Pb} 6 \mathrm{p}$ states dominate. In total, the broad structure extending from the Fermi level and up to around 20-25 eV consists of $\mathrm{Pb} 6 p$ and $6 d$ states, and chalcogen $p$ and $d$ states, in about equal amounts. In the low-energy part of this structure, the $p$ states dominate as already mentioned, whereas the $d$ states are concentrated to the energy region above $5 \mathrm{eV}$. In the region around 4-5 eV above the Fermi level two rather sharp peaks can be observed in the state density of all three compounds. The larger of these peaks is observable in the $p$ and $d$ partial state densities of both $\mathrm{Pb}$ and the chalcogenide. Flat bands in this energy region, corresponding to the peaks, can be found in the $[X \Gamma]$ part of the band structures in Figure 3 and in the diamond-shaped faces of the Brillouin zone, i.e., those faces that have $X$ as 

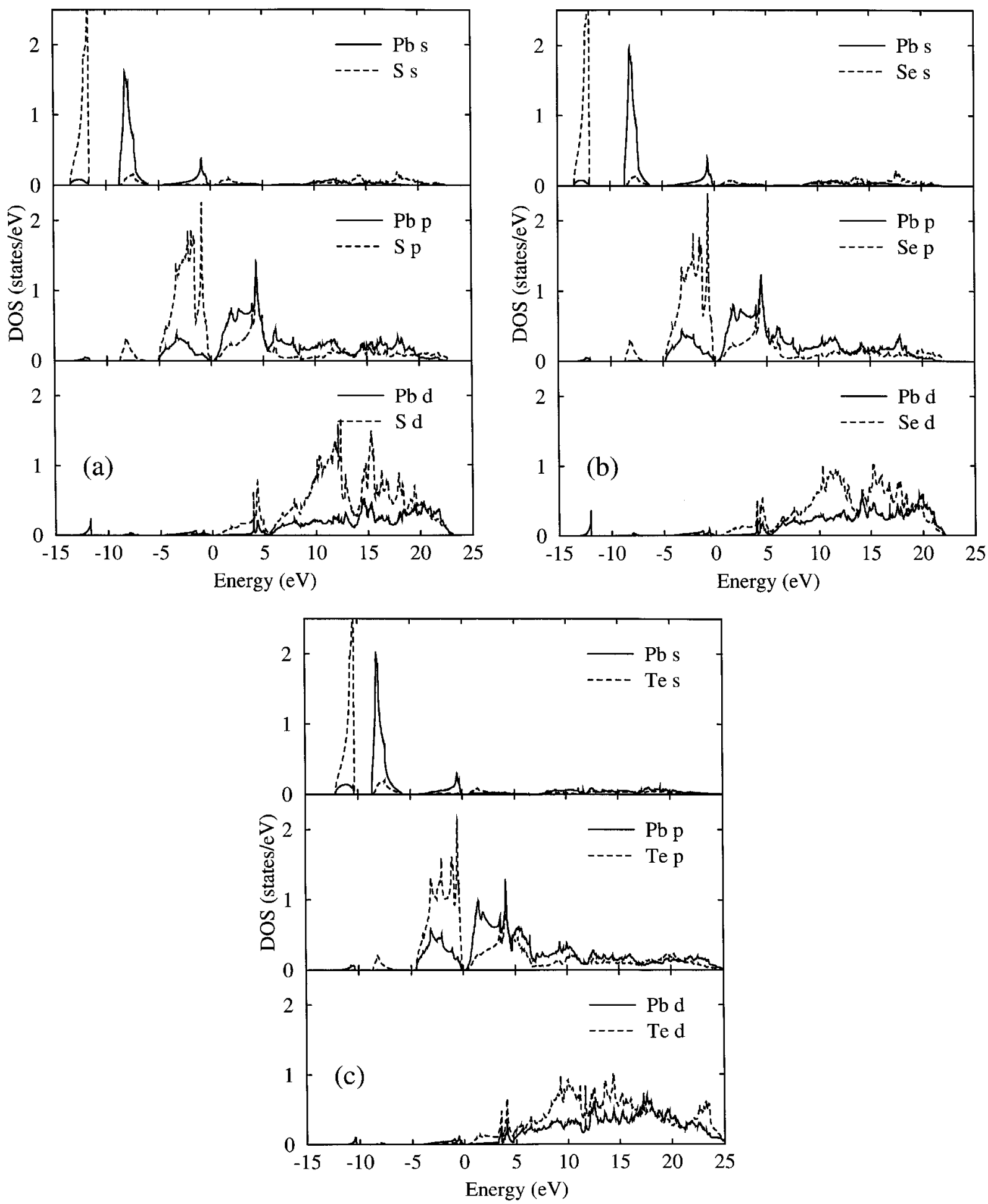

FIGURE 2. Partial $s, p$, and $d$ DOS (in states per electron volt and for (a) PbS, (b) PbSe, and (c) PbTe. The Fermi energy is at zero. 
DELIN ET AL.
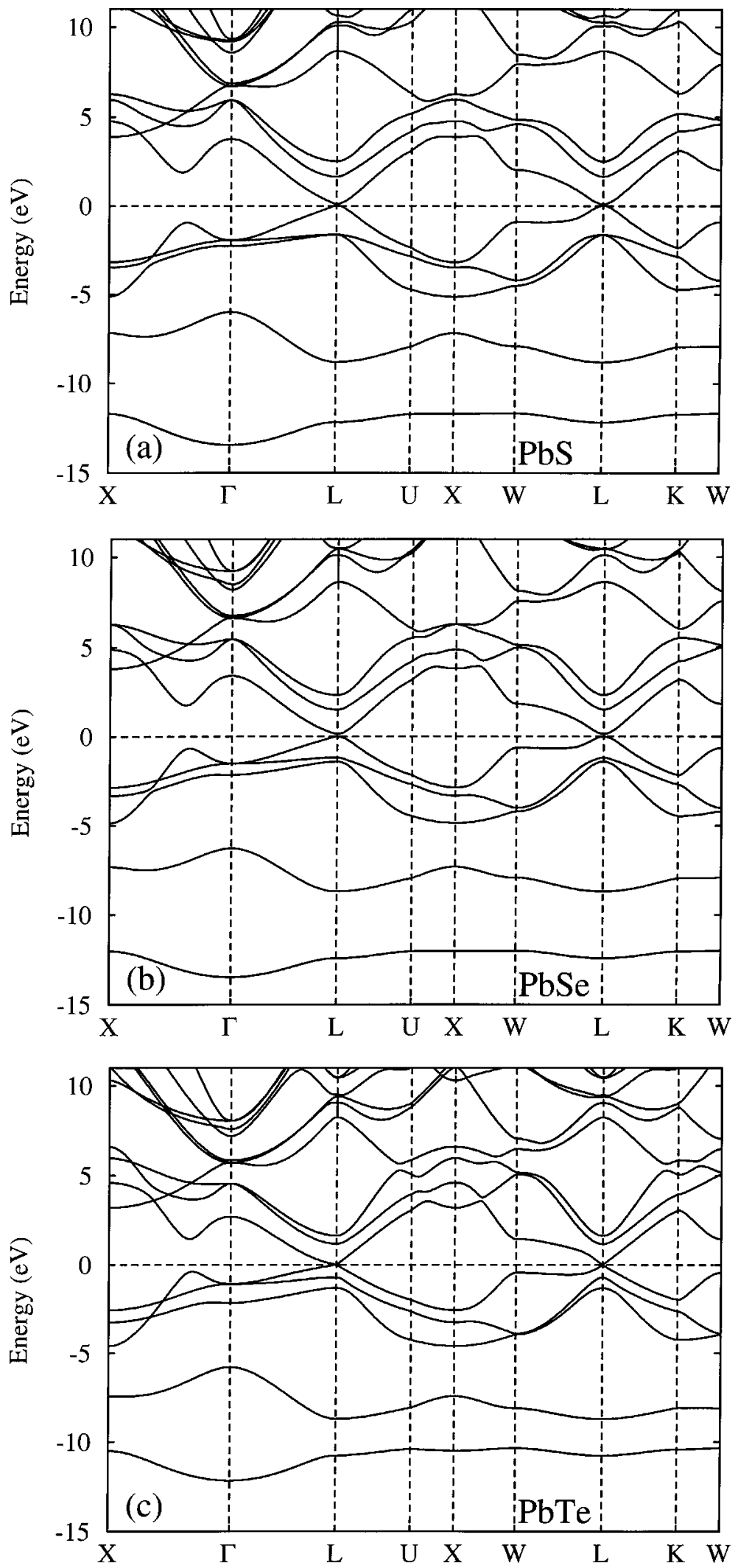

FIGURE 3. Band structure of (a) PbS, (b) PbSe, and (c) PbTe along high symmetry directions in the Brillouin zone. The Fermi energy is at zero. 
central point. Distinctive structures in the DOS above $5 \mathrm{eV}$ are one broad peak at around $12 \mathrm{eV}$ and another family of higher peaks at $15 \mathrm{eV}$, at least in PbS and PbSe. These structures come predominantly from the chalcogen $d$ states. For $\mathrm{PbTe}$, however, this part of the DOS is rather featureless.

From the partial state densities, it is clear that the main bonding mechanism in the lead salts is hybridization between the $\mathrm{Pb} 6 p$ states and the chalcogen $p$ states. The bond is both ionic and covalent in character, covalent since the $\mathrm{Pb} 6 p$ states and the chalcogenide $p$ states are strongly hybridized and degenerate over a large part of their extension, and ionic since the relative amount of $\mathrm{Pb} 6 p$ states and chalcogen $p$ states is different above and below the Fermi level. Below the Fermi level, the chalcogen $p$ states dominate, whereas above, the $\mathrm{Pb} 6 p$ states dominate. From simple argumentation using the relative electronegativity of the chalcogen and $\mathrm{Pb}$, we expect that $\mathrm{PbS}$ should be the most ionic and PbTe the most covalent of the three systems since $\mathrm{Pb}$ has an electronegativity (according to Pauling) of 1.8, and S, Se, and Te have electronegativities 2.5, 2.4, and 2.1, respectively. This conclusion compares well with what we expect from Figure 2. The inmixing of $\mathrm{Pb} 6 p$ states below the Fermi level increases from $\mathrm{PbS}$ to $\mathrm{PbTe}$, which corresponds to making PbTe the most covalent of these compounds and $\mathrm{PbS}$ the most ionic. A more quantitative analysis of this can be done by integrating the partial state densities, giving the number of states inside each muffin-tin sphere. However, it is important to be aware of that the absolute numbers will depend on the absolute and relative sizes of the muffin-tin spheres. In the present calculation, the $\mathrm{Pb}$ sphere and the chalcogenide sphere are of equal size and almost touching for each compound. Thus, the absolute size of the spheres in PbTe is larger than in $\mathrm{PbS}$ since the lattice parameter is larger in the former compound, and the sphere size does not correspond to the atomic radii of the elements. Integration of the site-decomposed DOS reveals that in $\mathrm{PbS}$ there are about 4 more valence electrons in the chalcogenide sphere than in the $\mathrm{Pb}$ sphere. In $\mathrm{PbTe}$, this number is reduced to 2.6. The main contribution to the difference between these two numbers comes from the $p$ states (1.1 electrons), with a small contribution from the $d$ states (0.3 electrons) and a negligible contribution from the $s$ states. Note, however, that since the sulfur atom is smaller than the tellurium atom, the above numbers exaggerate somewhat the difference in ionicity between the $\mathrm{PbS}$ and $\mathrm{PbTe}$.

Finally, it is interesting to compare our calculated gaps with experimental data. Since quasiparticle excitations are not taken into account in density functional theory (DFT), the energy gap calculated from DFT, often called the Kohn-Sham gap, tends to be smaller than the experimental one. In some cases, even the wrong ground state is predicted, as, e.g., in Ge, where the energy gap is around $0.7 \mathrm{eV}$, whereas the LDA Kohn-Sham gap is slightly negative at ambient pressure [24]. Experimentally, it is well known that the lead salts have a narrow, direct gap at the $L$ point [25]. This is also what we find. At liquid helium temperature, the experimental energy gaps are 0.286, 0.165, and $0.19 \mathrm{eV}$ for $\mathrm{PbS}, \mathrm{PbSe}$, and $\mathrm{PbTe}$, respectively [26]. At ambient temperature, i.e., the temperature used in the ellipsometry measurements, the energy gaps are larger: 0.41 for $\mathrm{PbS}, 0.27$ for $\mathrm{PbSe}$, and 0.31 for PbTS [26]. In our DFT calculations we have obtained direct Kohn-Sham energy gaps of 0.069 $\mathrm{eV}$ for $\mathrm{PbS}, 0.141 \mathrm{eV}$ for PbSe, and $0.032 \mathrm{eV}$ for $\mathrm{PbTe}$.

Our theoretically calculated absorptive part of the dielectric functions for $\mathrm{PbSe}$ and $\mathrm{PbTe}$ are shown and compared to experimental spectra [7-9] in Figure 4. For PbS, several measurements of the reflectivity have been published, but as yet no ellipsometry measurement has been performed. Of course, we could have chosen to compare our calculated spectra also with results from reflectivity measurements. Our reasons for not doing so are as follows. Obviously, the dielectric functions obtained from reflectivity measurements and from ellipsometry differ in important respects. Compare, for instance, the absolute amplitudes of the dielectric functions measured with ellipsometry [7, $8]$ to the dielectric functions calculated from reflectivity experiments [13, 27]. First of all, the latter amplitudes are much smaller. For PbTe, Cardona and Greenway [27] report a maximum amplitude for $\epsilon_{2}$ of 25, and Kohn et al. [13] report a maximum amplitude of 7 , whereas Suzuki et al. [7, 8], using ellipsometry, find an amplitude close to 50 . The small amplitudes could be due to uncontrolled intensity losses in the reflectivity measurements, perhaps originating from surface roughness, or they could be due to the extrapolations necessary for the Kramers-Kronig transformation, as explained above. Maybe more important is the fact that the trend when changing chalcogen is opposite in the reflectivity measurements com- 


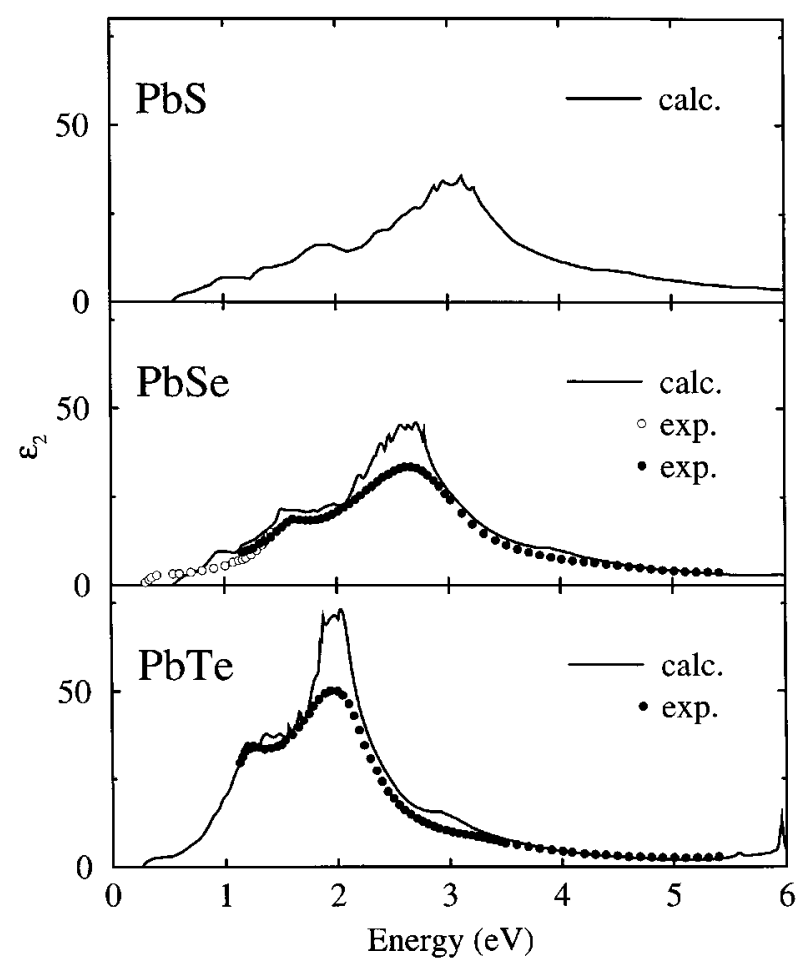

FIGURE 4. Calculated and experimental absorptive part of the dielectric function. The calculated spectra have not been broadened. The experimental spectra for $\mathrm{PbSe}$ are taken from Refs. [7] and [9], and the experimental spectrum for $\mathrm{PbTe}$ is from Ref. [8]. No ellipsometry measurement has yet been performed on $\mathrm{PbS}$.

pared to the ellipsometry measurements. Kohn et al. [13] find that the maximum amplitude of $\epsilon_{2}$ decreases from $\mathrm{PbS}$ to PbTe, whereas Suzuki et al. $[7,8]$ report a larger amplitude for $\mathrm{PbTe}$ than for $\mathrm{PbSe}$. We therefore conclude that, in order to obtain a meaningful comparison, it is important to use experimental results where the crystals have been built and surface treated in a similar fashion, and ideally also where the same experimental equipment and method has been used. Also, it seems that the experimental results reported in Refs. [7-9] for these systems are of superior quality. We base this conclusion mainly on the fact that these measurements report the highest amplitudes for $\epsilon_{2}$. Note, however, that the maxima of the spectra occur at the virtually same energies in all measurements made. We will make use of this fact below.

We now turn to an analysis of the optical spectra. The absorptive part of the dielectric function, $\epsilon_{2}$, is shown in Figure 4. The calculated spectra have been rigidly shifted in order to correct for the
DFT underestimation of the band gaps. Thus, $\epsilon_{2}$ has been shifted $0.3 \mathrm{eV}$ for PbS and PbSe, and 0.15 $\mathrm{eV}$ for PbTe. In this way, the maxima of our calculated $\epsilon_{2}$ coincide with the maxima of the measured spectra. The calculated spectra are not broadened, and thus have more structure than the experimental spectra. It is seen that $\epsilon_{2}$ is rather similar for all three lead salts. The main feature is a broad peak with maximum around $3 \mathrm{eV}$ for $\mathrm{PbS}$, $2.6 \mathrm{eV}$ for $\mathrm{PbSe}$, and $2 \mathrm{eV}$ for $\mathrm{PbTe}$. The maximum amplitude is almost twice as high for PbTe compared to $\mathrm{PbS}$. Also, for $\mathrm{PbTe}$ the main peak is much more narrow. In the experimental spectra in Figure 4 for PbSe and $\mathrm{PbTe}$, a shoulder around 1.5 $\mathrm{eV}$ is clearly visible. For PbS, no such shoulder is visible. The peak as well as the shoulder is excellently reproduced in the calculations, as are the general form of the spectra. The maximum amplitudes of the calculated spectra are significantly higher than in the experimental spectra. This is so because the calculated spectra are unbroadened. In order to compare amplitudes of experimental and calculated spectra, it is better to compare integrals of the spectra instead of maximal amplitudes. If we do this, we find that also the amplitudes are excellently reproduced in our calculations. Broadening is probably also the reason why the bumps at $4 \mathrm{eV}$ in the $\mathrm{PbS}$ spectrum and $3 \mathrm{eV}$ in the $\mathrm{PbTe}$ spectrum are not visible in the experimental spectra. Our calculated spectra are also very similar to the spectra in Ref. [13], which were obtained with the empirical pseudopotential method.

The trends in $\epsilon_{2}$ as a function of chalcogen may be linked to the trends observed in the DOS and band structures. Compare the highest lying valence bands (HVB) for the three systems and also the lowest lying conduction bands (LCB). Our band-resolved optical calculations show that transitions between these two bands account for almost all structure in the optical spectra at energies below $6 \mathrm{eV}$. In PbTe, these bands have clearly less dispersion than in PbS. This is the reason why the main peak moves to lower energy and becomes sharper as the chalcogen column is traversed downward. From the partial state densities in Figure 2 , it is rather obvious that the $p$ states play a major role in these optical transitions, both as initial and final states. Of the $s$ states, the $\mathrm{Pb}$ states primarily serve as initial states, whereas the chalcogen states are mostly final states. The $\mathrm{Pb}$ and chalcogen $d$ states are primarily final states.

Optical transitions between bands that are parallel or nearly so in an appreciable part of the BZ 
tend to result in peaks in the optical spectrum. The HVB and LCB in our calculations are in fact quasi parallel in appreciable regions on and around the Jones zone plane [28], which for systems with face-centered cubic (fcc) symmetry and 10 valence electrons per unit cell includes the $\mathbf{k}$ points $W, L$, and $\left(\frac{1}{4} \frac{1}{4} 0\right)$. Transitions in this region account for both the main peak as well as the shoulder. Generally, the Jones zone is defined as the higher order $\mathrm{BZ}$ in the extended zone scheme that exactly contains all the occupied Bloch states. The basic idea behind the concept of the Jones zone, then, is that it is a simple way of finding the planes which are most cut by the free electron Fermi sphere. The Jones zone bands between $W$ and $L$ are visible in Figure 3.

Next, we consider the dispersive part of the dielectric function, $\epsilon_{1}$, for the three lead salts; see Figure 5. The calculated spectra have been obtained by Kramers-Kronig transformation of the shifted $\epsilon_{2}$ spectra. The main features are a shoulder at lower energies, a rather steep decrease between 2 and $3 \mathrm{eV}$, after which $\epsilon_{1}$ becomes nega-

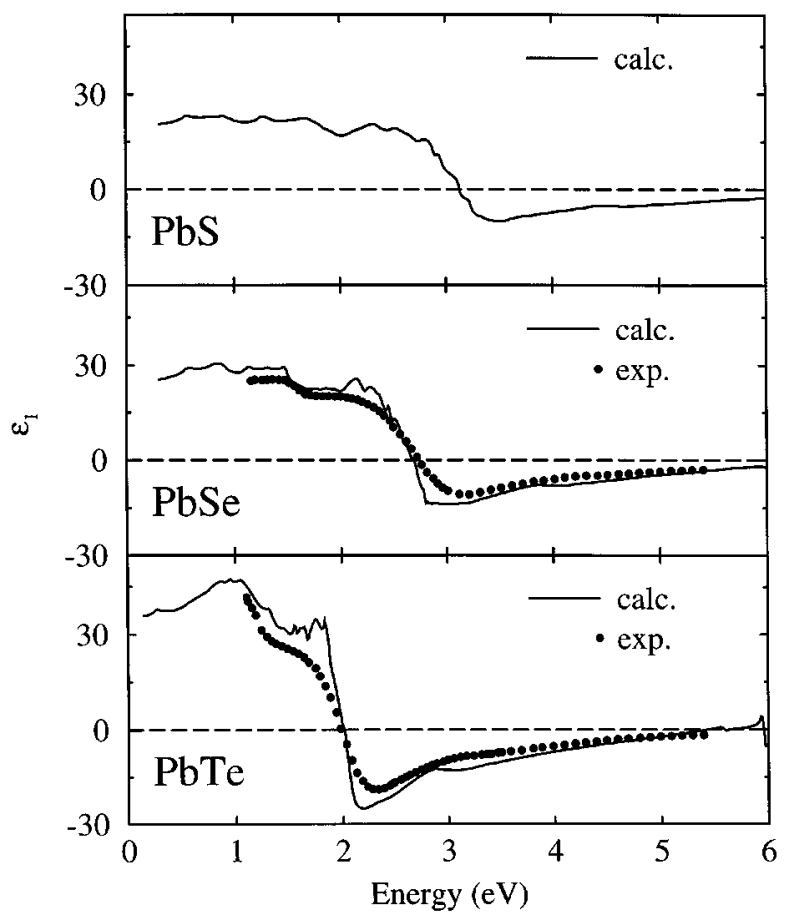

FIGURE 5. Calculated and experimental dispersive part of the dielectric function. The calculated spectra have not been broadened. The experimental spectrum for $\mathrm{PbSe}$ is taken from Ref. [7], and the experimental spectrum for PbTe is from Ref. [8]. No ellipsometry measurement has yet been performed on $\mathrm{PbS}$. tive, a minimum, and then a slow increase toward zero at higher energies. All these features are very well reproduced in the calculated spectra. The structures are more pronounced in the calculated spectra since these are not broadened.

Finally, we shortly discuss some of the spectra obtainable from the dielectric function. Neither of these spectra are shown here. As seen in Figure 5, $\epsilon_{1}$ is zero at 3.1, 2.7, and $2.0 \mathrm{eV}$ for PbS, PbSe, and $\mathrm{PbTe}$, respectively. In $k$, i.e., the imaginary part of the refractive index, we find local maxima at these energies. On the contrary, in the energy loss spectra, no maximum is present at these energies, since $\epsilon_{2}$ is still large at these energies. Higher up, however, at around $14 \mathrm{eV}$, all three lead salts show a large peak in the energy loss spectrum. This peak corresponds to $\epsilon_{1}$ going through zero once again, this time becoming positive. At such high energies $\epsilon_{2}$ is small, and thus the amplitude of the energy loss may become large.

\section{Conclusions}

In conclusion, we have shown that the optical properties of the lead salts $\mathrm{PbS}, \mathrm{PbSe}$, and $\mathrm{PbTe}$ are excellently reproduced using density functional theory, if we allow for a rigid shift of the band structure, the so-called scissors operator. Not only the general form but also the absolute amplitudes are very well reproduced in our calculations. The agreement on the amplitudes, which has not been obtained before, is not so much a result of better calculational methods but is mainly due to improved experimental techniques. Furthermore, we have identified the microscopic origin of the main features in the optical spectra and found that transitions between the highest lying valence band and the lowest lying conduction band are responsible for most of the optical absorption in these systems. These bands are quasi-parallel on and in the vicinity of the (311) plane in the BZ, and thus it is this region of the $\mathrm{BZ}$ that is the most interesting as regards optical properties of the lead salts.

\section{ACKNOWLEDGMENTS}

The authors are grateful to the Swedish Research Council for Engineering Sciences, the Swedish Natural Science Foundation for financial support. We also wish to thank the Swedish Materials Consortium No. 9. 


\section{References}

1. R. Dalven, in Solid State Physics, Vol. 28, H. Ehrenreich, F. Seitz, and D. Turnbull, Eds. (Academic, New York, 1973), 179.

2. R. A. Cowley, Philos. Mag. 11, 673 (1965); K. Murase, J. Phys. Soc. Jpn. Suppl. 49, 725 (1980).

3. See, e.g., G. P. Agrawal and N. K. Dutta, Semiconductor Lasers (Van Nostrand Reinhold, New York, 1993), p. 547.

4. L. D. Hicks, T. C. Harman, X. Sun, and M. S. Dresselhaus, Phys. Rev. B 53, R10493 (1996).

5. S. Chatterjee and U. Pal, Optical Eng. 32, 2923, (1993); T. K. Chaudhuri, Int. J. Energy Res. 16, 481 (1992); M. T. Mohammad, Sol. Energy Mat. 20, 297 (1990).

6. P. K. Nair, M. Ocampo, A. Fernandez, and M. T. S. Nair, Sol. Energy Mat. 20, 235 (1990); P. K. Nair, A. Fernandez, and M. T. S. Nair, Proc. SPIE Int. Soc. Opt. Eng. 1149, 88 (1989).

7. N. Suzuki, K. Sawai, and S. Adachi, J. Appl. Phys. 77, 1249 (1995).

8. N. Suzuki and S. Adachi, Jpn. J. Appl. Phys. 33, 193 (1994).

9. T. R. Globus, A. O. Olesk, and S. A. Olesk, Sov. Phys. Semicond. 24(1), 22 (1990).

10. A. Delin, O. Eriksson, R. Ahuja, B. Johansson, M. S. S. Brooks, T. Gasche, S. Auluck, ard J. M. Wills, Phys. Rev. B 54, 1673 (1996).

11. A. Delin, P. Oppeneer, M. S. S. Brooks, T. Kraft, J. M. Wills, B. Johansson, and O. Eriksson, Phys. Rev. B 55, R10173 (1997).

12. G. Lehmann and M. Taut, Phys. Stat. Sol. (B) 54, 469 (1972); P. J. Lin and L. Kleinmann, Phys. Rev. 142, 478 (1966); Y. W
Tang and M. L. Cohen, Phys. Rev. 180, 823 (1969).

13. S. E. Kohn, P. Y. Yu, Y. Petroff, Y. R. Shen, Y. Tsang, and M. L. Cohen, Phys. Rev. B, 8, 1477 (1973).

14. J. B. Conkin, L. E. Johnson, and G. W. Pratt, Phys. Rev. 137, A1282 (1965); S. Rabii, Phys. Rev. 167, 801 (1968); D. D. Buss and N. J. Parada, Phys. Rev. B, 1, 2692 (1970).

15. F. Herman, R. L. Kortum, I. B. Ortenbarge, and J. P. Van Dyke, J. Phys. (Paris) Suppl. 29, C4-62 (1968).

16. C. S. Lent, M. A. Bowen, J. D. Dow, R. S. Allgaiger, O. F. Sankey, and E. S. Ho, Superlatt. Microstruct. 2, 491 (1986).

17. J. A. Valdivia and G. E. Barberis, J. Phys. Chem. Solids, 56, 1141 (1995).

18. P. Hohenberg and W. Kohn, Phys. Rev. 136, B864 (1964); W. Kohn and L. J. Sham, Phys. Rev. 140, A1133 (1965).

19. J. M. Wills (unpublished); J. M. Wills, and B. R. Cooper, Phys. Rev. B 36, 3809 (1987); D. L. Price and B. R. Cooper, Phys. Rev. B 39, 4945 (1989).

20. J. P. Perdew, in Electronic Structure of Solids 1991, P. Ziesche and H. Eschrig, Eds. (Akademie, Berlin, 1991), Vol. 11, see also http://camchem.rutzers.edu/ kieron/dft_pubs. html.

21. O. K. Andersen, Phys. Rev. B 12, 3060 (1975); H. L. Skriver The LMTO Method (Springer, Berlin, 1984).

22. D. J. Chadi and M. L. Cohen, Phys. Rev. B 8, 5747 (1973); S. Froyen, Phys. Rev. B 39, 3168 (1989).

23. R. Ahuja, S. Auluck, J. M. Wills, M. Alouani, B. Johansson, and O. Eriksson, Phys. Rev. B 55, 4999 (1997).

24. M. Alouani and J. M. Wills, Phys. Rev. B 54, 2480 (1996).

25. R. Dalven, Infrared Phys. 9, 141 (1969).

26. W. H. Strehlow and E. L. Cook, J. Phys. Chem. Ref. Data 2, 163 (1973).

27. M. Cardona and D. L. Greenway, Phys. Rev. 133, 1685.

28. V. Heine and R. O. Jones, J. Phys. C 2, 719 (1969); Y. Onodera, Sol. St. Comm. 11, 1397 (1972). 